\title{
STATIC PERSON DETECTION AND LOCALIZATION BASED ON THEIR RESPIRATORY MOTION USING VARIOUS ANTENNA TYPES
}

\author{
Daniel NOVÁK, Ján SCHNEIDER, Dušan KOCUR \\ Department of Electronics and Multimedia Communications, Faculty of Electrical Engineering and Informatics, \\ Technical University of Košice, Letná 9, 04200 Košice, Slovak Republic, Tel.: +421 55602 4234, \\ E-mail:daniel.novak@tuke.sk, jan.schneider@tuke.sk,dusan.kocur@tuke.sk
}

\begin{abstract}
Detection and localization of non-moving human targets by ultra-wideband radars (sensors) is becoming crucial in the last years. In contrast to a moving target localization this task is much more challenging. A movement of a static person caused by the respiratory motion is barely observable. In this paper, a new method of respiratory motion detection based on Welch periodogram is presented. The performance of this method is demonstrated on a scenario with real radar data obtained by a measurement at laboratory conditions. Moreover, a custom-made flat antipodal Vivaldi antennas are used. Despite of the conditions, one static person is succesfully detected and localized.
\end{abstract}

Keywords: UWB radar, static person, respiratory motion, Welch periodogram, antipodal Vivaldi antenna, person detection and localization

\section{INTRODUCTION}

Ultra-wideband (UWB) radars are progressive devices used for various type of nonintrusive monitoring of people movement. The term nonintrusive means that a radar can operate without any awareness of the monitored persons. UWB radar can be used in many applications for human surveillance e.g. monitoring of elder people [1], moving person monitoring [2] or human vital signs monitoring [3]. A special attention is given to the moving targets monitoring due to the increasing terrorist threads in the recent years.

Usually, the signals emitted by an UWB radar have an ability to penetrate through the solid non-metallic obstacles and back [4]. Therefore, using proper signal processing methods, we are able to detect, localize and track various motions belonging to the human targets. In the actual research state, every type of human movement (e.g. walk, respiratory motions, heartbeat etc.) has to be processed with its own set of signal processing methods. Therefore, methods used for moving target surveillance (presented in [2]) do not operate properly in a situation when a monitored target remains on his/her place. Such human target is referred to as a static person.

A static person appears as a clutter in comparison with a moving person and is much harder to detect. In this paper, we propose a method that is able to detect and even localize a static person based on his/her respiratory motions. For this purpose, an UWB radar device with three antennas (one transmitting (Tx) and two receiving ( $\mathrm{Rx}$ ) antennas) will be used.

The proposed method of the static person detection consists of a signal power spectrum calculation followed-up by the application of a two-stage detector. The power spectrum is calculated by Welch periodogram method [5]. The first detection stage consists of usage of the Order Statistics Constant False Alarm detector with guarding interval (OS-CFAR) [6]. The second detector stage consists of a treshold detector using constant treshold value. Eventually, a localization of the target can be performed.

Within this paper, two similar measurements were per- formed in order to compare the performance of two antenna types. Within our previous laboratory experiments, we used the double ridged horn antennas RFspin DRH10 with frequency range $0.74 \mathrm{GHz}-10.5 \mathrm{GHz}$, dimensions (width $\times$ length $\times$ depth) $148 \mathrm{~mm}$ x $204 \mathrm{~mm}$ x $242 \mathrm{~mm}$ and weight around $1.24 \mathrm{~kg}$. The second antenna type are the low-cost antipodal Vivaldi antennas with low profile, originally introduced in [7]. These antennas are printed on ARLON 600 substrate with relative permitivity $\varepsilon_{r}=$ 6.15 and tangent loss $\tan _{\delta}=0.003$. The top layer of the antenna feeds the signal conductor and the bottom layer feeds the ground plane of the feeding line. Final dimensions of the antenna are (width $\mathrm{x}$ length $\mathrm{x}$ height) $128 \mathrm{~mm} \times 190 \mathrm{~mm} \times 1.575 \mathrm{~mm}$ and weight only $0.108 \mathrm{~kg}$. The antennas operate in the frequency band from $0.81 \mathrm{GHz}$ to $12 \mathrm{GHz}$ with the average gain $6.32 \mathrm{dBi}$, fractional bandwidth $\mathrm{BW}=163 \%$ and with the low ringing factor. A prototype of the manufactured antenna is shown in Fig. 1. Antenna pattern for the frequency $2 \mathrm{GHz}$ and $4 \mathrm{GHz}$ are shown in Fig. 2 and Fig. 3 respectively.

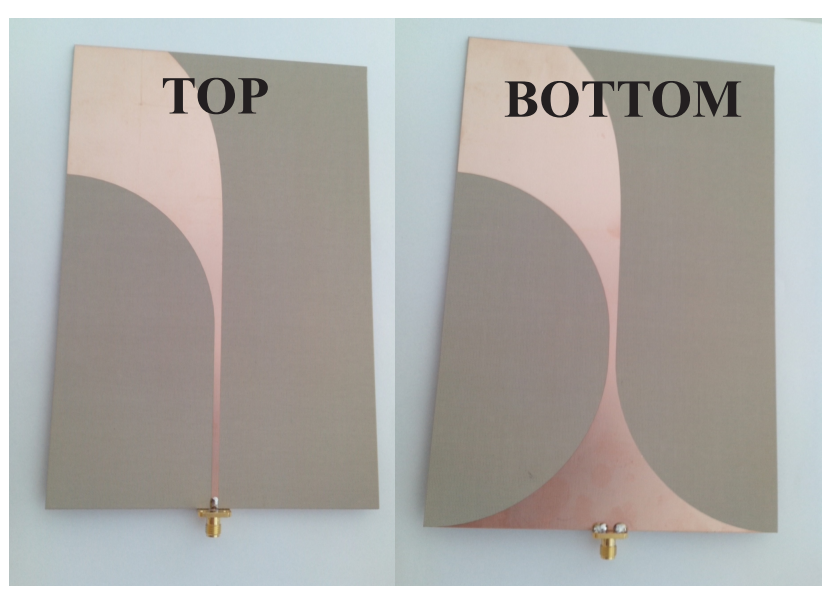

Fig. 1 Antipodal Vivaldi antennas 


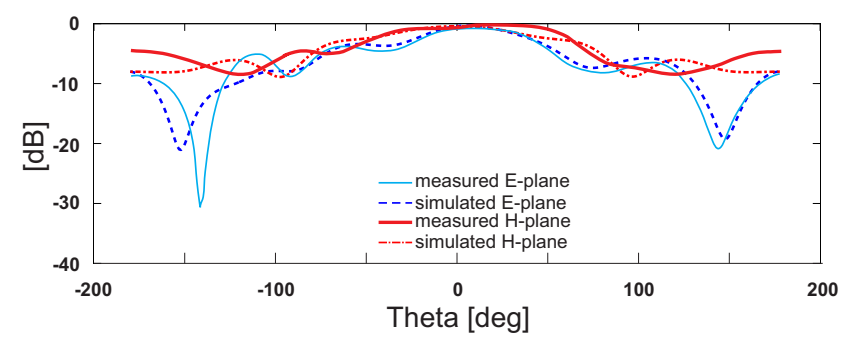

Fig. 2 Antenna pattern of the planar antipodal Vivaldi antenna at $2 \mathrm{GHz}$

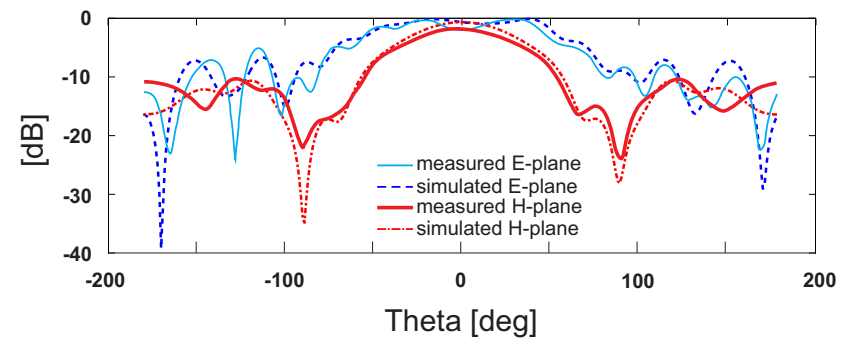

Fig. 3 Antenna pattern of the planar antipodal Vivaldi antenna at $4 \mathrm{GHz}$

This paper is organized as follows. Section 2 provides the description of the respiratory motion detection. The third section describes the novel respiratory motion detection method. Section 4 shows the results of this method using the radar signals obtained from a laboratory measurement. In the conclusion, method performance is evaluated.

\section{RESPIRATORY MOTION}

The respiratory motion can be described as a movement of a person's chest which is performed during the breathing process. The identification of the respiratory motion in environment can be described as follows. An antenna converts an input signal $p(t)$ into an electromagnetic waves which propagate through a monitored area. The emitted signal is partially reflected back to the antenna and converted by a radar into an impulse response of the environment $h(t, \tau)$. Usually, a static human target is situated behind a solid nonmetallic obstacle (e.g. wall) at a certain distance $d_{0}$. The static person is exposed to the UWB signal emitted by the radar antenna. For simplicity, we can assume that the transmitting and receiving antennas (Tx and $\mathrm{Rx}$ ) are represented by the same antenna while the electromagnetic wave emitted by the Tx propagates through the front wall and by a multipath environment to the Rx. The emitted signal is partially reflected from the body of the person and it travels back, where is captured by the Rx antenna. The radar device converts the received signals using a pulse compression method into an impulse response of the environment $h(t, \tau)$ as follows:

$$
\begin{aligned}
h(t, \tau) & =\sum_{i=1}^{N} A_{i} p\left(t-t_{i}\right)+A_{0} p\left(t-t_{d}(\tau)\right) \\
& =h_{b}(t, \tau)+h_{0}(t, \tau)
\end{aligned}
$$

where $h_{b}(t, \tau)$ is part of the signal representing the static background (clutter) and $h_{0}(t, \tau)$ represents a signal component that are due to the static target. Constant term $A_{i}$ in (1) denotes the path gain or loss of the $i$-th signal path. The time variable $t_{d}(\tau)$ takes values according to the distance of the target $\left(d_{0}\right)$ from the antenna. The time $t_{d}(\tau)$ is referred to as time-of-arrival (TOA). TOA expresses the time interval which electromagnetic wave needs to propagate along the Tx-target-Rx trajectory. The movement of the person's chest caused by the respiratory motions are observable as a slight and periodic changes of the TOA within the received radar data [8]. TOA is expressed as

$t_{d}(\tau)=2 d\left(t_{0}, \tau\right) / c$

where

$d\left(t_{0}, \tau\right)=d_{0}\left(t_{0}, \tau\right)+m(\tau)=d_{0}\left(t_{0}, \tau\right)+m \sin (2 \pi f \tau)$

The term $d_{0}\left(t_{0}, \tau\right)$ is a DC component expressing the reflection from a static human target situated at a distance $d_{0}$ from the radar antenna. On the other hand, the second part of (3) represents an alteration of the person's chest position caused by the respiratory motion. Its magnitude $m$ usually takes values between $0.2 \mathrm{~cm}$ and $0.6 \mathrm{~cm}$ [9]. After approx. 1-2 minutes of observation time, human breathing can be generalized as a periodic process with its own breathing frequency $f$. The frequency of the first harmonic component of the breathing rate usually takes values between $0.2 \mathrm{~Hz}$ and $0.7 \mathrm{~Hz}$ (e.g. [8,-10]).

The above mentioned comments summarizing the detailed analyses presented in [8] and [10] indicate, that a static person can be detected based on a detection of a periodical signal components (i.e. a periodical motion) with a frequency from the interval $0.2 \mathrm{~Hz}-0.7 \mathrm{~Hz}$ with regard to the slow-time variable $(\tau)$ for a constant fast-time instant $\left(t_{0}\right)$. For that purpose, the radar signal processing procedure for static person detection and localization described in the next section can be used.

\section{WELCH PERIODOGRAM METHOD}

The radar signal processing procedure for detection and localization of a static person consists from the following phases: radar calibration (raw radar signal preprocessing), background subtraction, weak signal enhancement, periodogram calculation, two-stage detection and target localization.

\subsection{Radar calibration (raw radar signal preprocess- ing)}

At the beginning, raw input signal $h(t, \tau)$ obtained by the radar is not calibrated. Due to the usage of a M-sequence radar, a starting chip of M-sequence is situated at a random position. Therefore, a constant round shift must be applied on every impulse response. This procedure is often called the time-zero setting. In case of using separate antennas for the Tx and $\mathrm{Rx}$, the most common method for the time-zero setting is the method using crosstalk between Tx and Rx [2]. Crosstalk is caused by transmitted electromagnetic wave from Tx directly into the Rx. 


\subsection{Background subtraction}

The calibrated signal contains some undesired signal components i.e. noise and stationary background $h_{b}(t, \tau)$. Static background is estimated and subtracted from the original calibrated signal $h(t, \tau)$. The background estimation is realized because the background itself (without any target) is unknown. Here, an estimation using exponential averaging method is applied [2].

\subsection{Weak signals enhancement}

Breathing itself is a barely observable movement. An amplitude change is very small so an improvement of a target echo to noise ratio is needed. To enhance the signal components appearing due to the respiratory motion, we need to separate the signal component $d\left(t_{0}, \tau\right)$ from the $h_{b}\left(t_{\text {const }}, \tau\right)$. The time instant $t=t_{\text {const }}$ stands for a constant value of the propagation time. Input signal is filtered by so-called slow-time filter (signal is filtered along the time axis $\tau$ ). As it was mentioned before, spectral components that are due to the human respiratory motion can be found within the interval $0.2 \mathrm{~Hz}-0.7 \mathrm{~Hz}$. A slow-time filter can be realized either as a bandpass filter with mentioned cutoff frequencies or as a lowpass filter with the upper cutoff frequency $(0.7 \mathrm{~Hz})$.

\subsection{Power spectrum estimation}

For estimation of the energy distribution into the signal spectrum, a periodogram can be used. Welch periodogram computes the power spectrum [5] from the signal components $s(t, \tau)=d\left(t_{0}, \tau\right)$.Here, the power estimates are computed for each row of the input signal. As we are searching for a static target, the power integration between frequencies of $0.2 \mathrm{~Hz}-0.7 \mathrm{~Hz}$ is performed in order to highlight the spectral components on the mentioned interval. In this signal $h_{W}(t, \tau)$ the power is concentrated on the TOA that corresponds to the real target position.

\subsection{Detection}

Presence of the target within the filtered signals is examined by the detector. At the detector input, the signal is treated as a testing (decision) statistics $X(t, \tau)$ and it is compared to the detector treshold $\gamma\left(t_{\text {const }}, \tau\right)$ as follows:

$h_{d}(t, \tau)= \begin{cases}0 & \text { if } X(t, \tau) \leq \gamma(t, \tau) \\ 1 & \text { if } X(t, \tau)>\gamma(t, \tau)\end{cases}$

Within this method, a two-stage detection is used. The first stage contains an adaptive OS-CFAR detector, the second stage uses a treshold detector.

\section{Adaptive detector}

Basic block diagram of the OS-CFAR detector with guarding interval is shown in Fig. 4 Since the background noise varies over a large timescales, an OS-CFAR detector is used to provide an adaptive detector treshold $\gamma(t, \tau)$ for each time instant $t$.
Basic operation of the OS-CFAR detector can be described as follows. As a testing statistics $X(t, \tau)$, signal of integrated power spectrum $h_{W}(t, \tau)$ is used. Within this signal a static human target is represented by multiple samples. Thus, guarding interval ensures that only samples of background and noise take into account. The rest of the signal samples in the reference window $N$ are sorted in ascending order by the size of their magnitude. The detector theshold is then estimated as

$\gamma(t, \tau)=h_{W}\left(t, \tau_{k}\right)$

where value $k$ is according to [11] set to $3 / 4$ size of sliding range window. It can be considered as a $3 / 4$ of a clutter power. Computation of a scale facror and more detailed description of the OS-CFAR detector is beyond this publication and it can be found in [11].

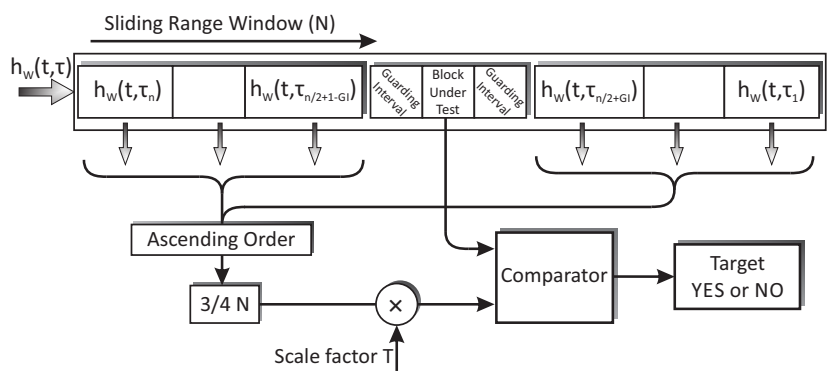

Fig. 4 OS-CFAR detector scheme

\section{Treshold detector}

Despite of the detector performance, a standalone false alarms may occur at the OS-CFAR detector output. As it was mentioned before, a static human target generates more than one echo (sample) at the received input signal. Such target is referred to as distributed target. Hence, integration of nearby samples is performed in order to join all nearby positive detections from the target into the one with bigger value. Intergated false alarms should not get value set as a detector theshold and, thus, they are not taken into account.

\section{EXPERIMENTAL RESULTS}

Method performance was tested on the radar data acquired in a laboratory measurement. It is a scenario, where a single static person is situated in front of the antennas behind a solid obstacle. The only observable movement was uplift and descent of his chest. In order to compare the performance of two types of antennas, two similar measurements were performed. In both cases, a person was sitting behind a solid obstacle. Measurement setup is shown in Fig. 5a.

Base of the co-ordinates system was the Tx antenna at position $[0 ; 0] \mathrm{m}$. The static person was sitting on a chair, but due to the different antenna stands, his position in two measurements was slightly different. In the first case, using the antipodal Vivaldi antennas, the target was situated at co-ordinates $[0.25 ; 2.3] \mathrm{m}$ from the Tx antenna (Fig. 5b). In the second case, using the double ridged horn antennas, his co-ordinates were $[0.5 ; 2.7] \mathrm{m}$ from the Tx (Fig. 5c). 


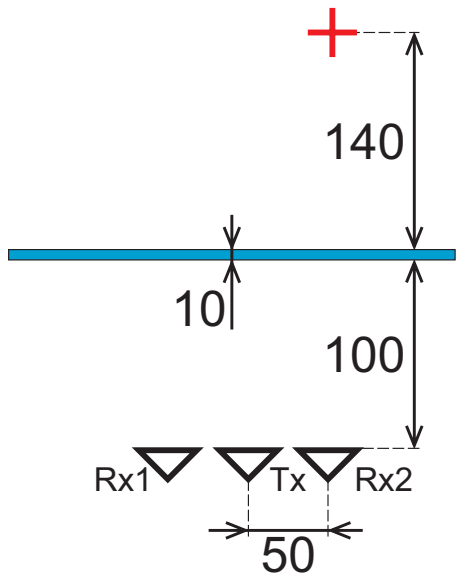

(a)

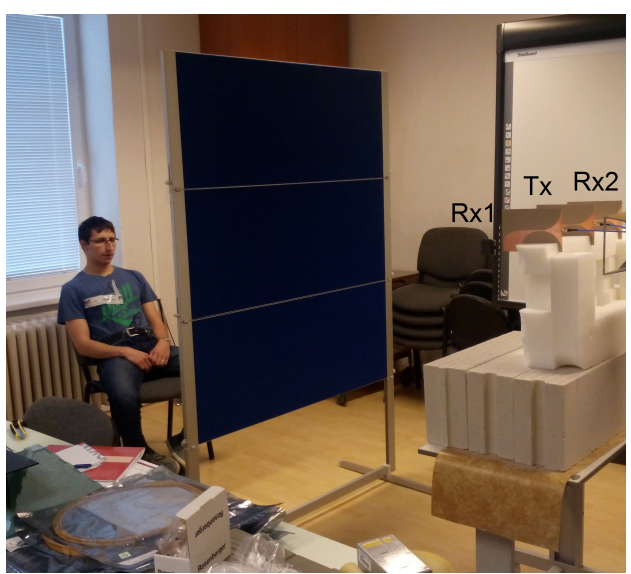

(b)

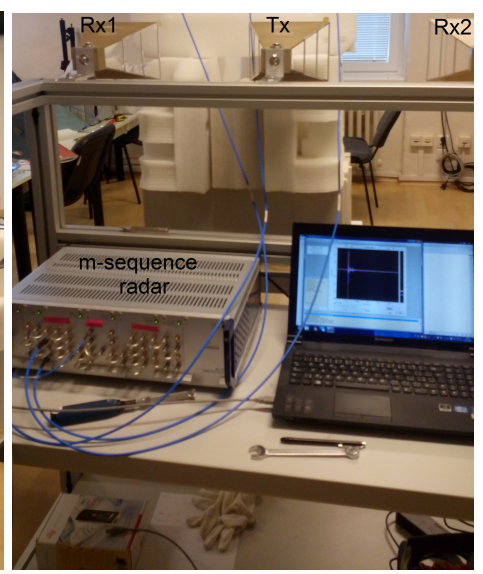

(c)

Fig. 5 Monitored area; (a) measurement setup (dimensions are in $\mathrm{cm}$ ), (b) (c) radar system and antenna configuration

Person was illuminated by the signal from the M-sequence UWB radar with the following parameters: radar clock frequency $13.82 \mathrm{GHz}$, length of binary sequence 12 bits (4095 samples per impulse response), impulse response duration $296.3 \mathrm{~ns}$, maximum range $44.5 \mathrm{~m}$, range resolution 1 chip $=0.0185 \mathrm{~m}$, radiated power $-6.5 \mathrm{dBm}$. Received signals were not externally amplified due to the laboratory conditions. The signal amplification was performed by the internal circuits of the UWB radar. However, monitored area contains several metallic parts of furniture and surroundings.

At first, the background is estimated and then subtracted (Fig. 7a). Then, signal components resulting from the presence of the static target are enhanced (filtered) by the band-pass filter with the cutoff frequencies $0.1 \mathrm{~Hz}$ and $0.7 \mathrm{~Hz}$ (Fig. 7b). Then, power spectrum is computed using Welch periodogram (Fig. 7c). Here, the spectral components caused by the respiratory motion of the target are slightly visible. As we are interested only for the specific frequency interval, we sum up all the periodogram columns within the band $[0.1 \mathrm{~Hz}, 0.7 \mathrm{~Hz}]$ into one signal $h_{W}(t, \tau)$. This signal is shown in Fig. $7 \mathrm{~d}$ for Rx1 and Fig. $7 \mathrm{e}$ for Rx2.
In order to detect a target presence within the signal, the two-stage detection is used. At first, an OS-CFAR detector with guarding interval is applied. In Fig. $7 \mathrm{f}$ for Rx1 and Fig. $7 \mathrm{~g}$ for $\mathrm{R} \times 2$ is shown an adaptive treshold value (dashed red) computed from the input signal (solid blue). In order to reduce the presence of possible false alarms, an individual nearby peaks (representing one distributed target) are summed-up into one peak with a bigger value. Position of the every peak denotes the TOA that belongs to the static target. The second detection stage now eliminates all the single false alarms (Fig. 7h for Rx1 and Fig. 7i for Rx2).

At this point, the TOA for every target is estimated correctly and no false alarms are present. Now the TOAs out of both $\mathrm{Rx}$ are associated into pairs, where the true TOA of the target can be estimated. This can be done by using the trace connection method. Target is then localized using the direct calculation method. We assume that the estimated position can take place within $30 \mathrm{~cm}$ from the center position (black circle). This is due to the bigger dimensions of the target in comparison with one point which is estimated by the localization phase. Result of the localization phase for both measurements can be seen in Fig. 6

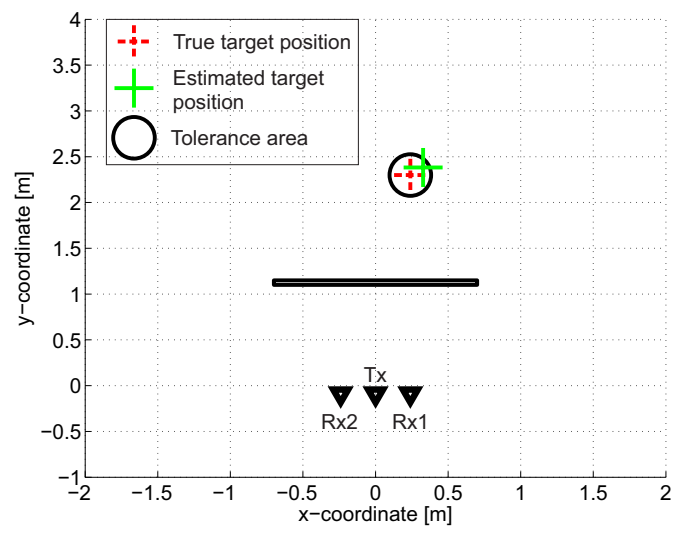

(a)

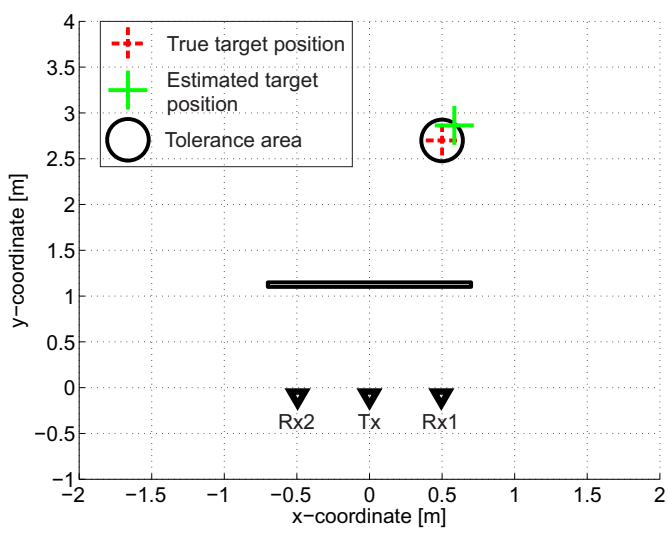

(b)

Fig. 6 Static person localization: (a) antipodal Vivaldi antennas, (b) double ridged horn antennas 


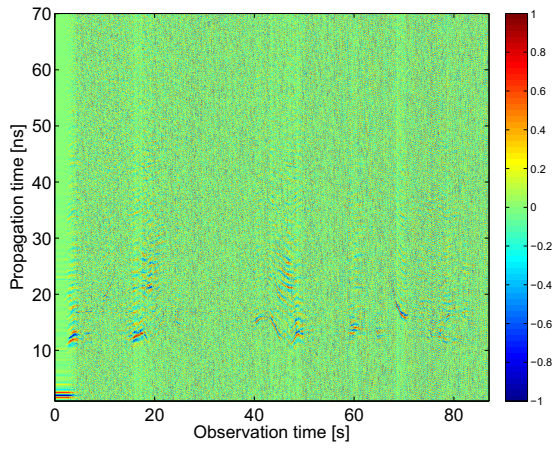

(a)

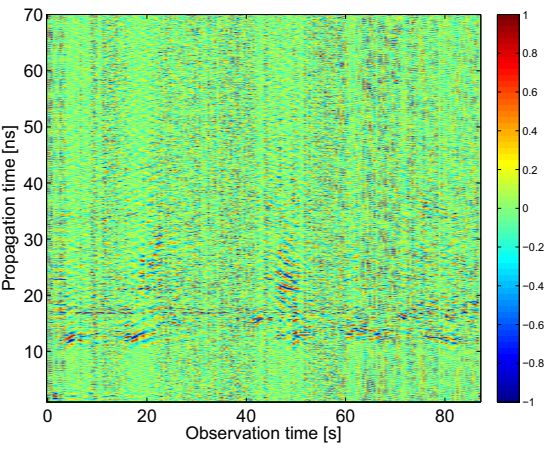

(b)

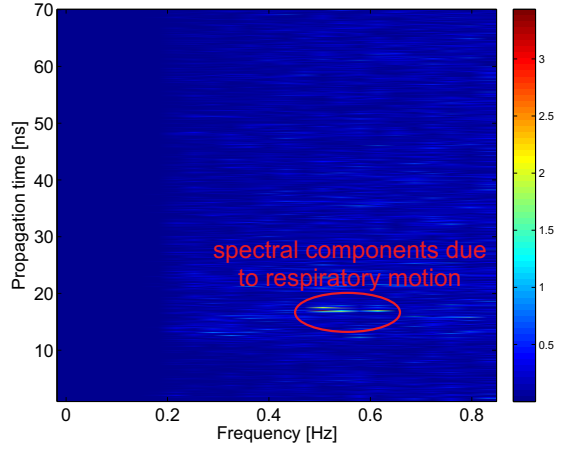

(c)

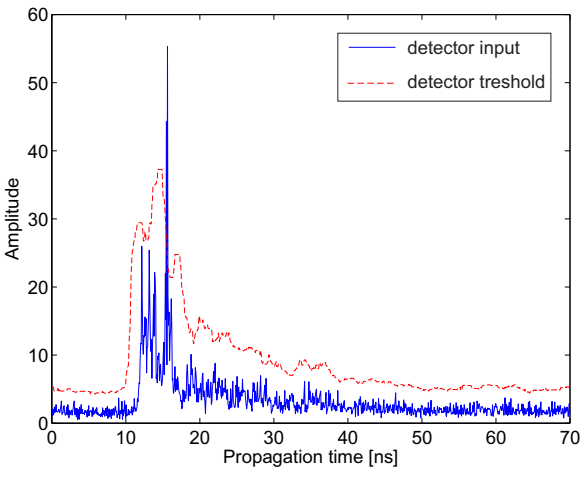

(d)

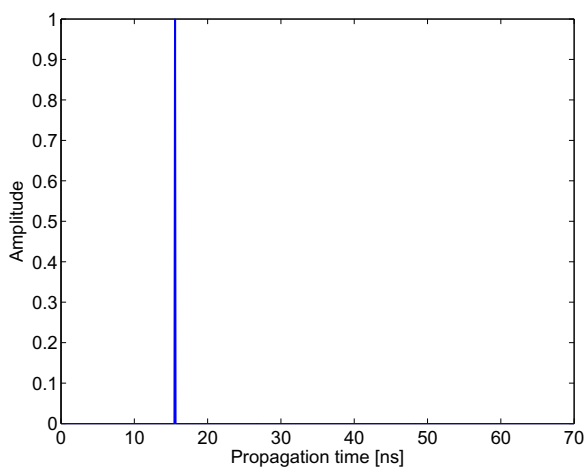

(f)

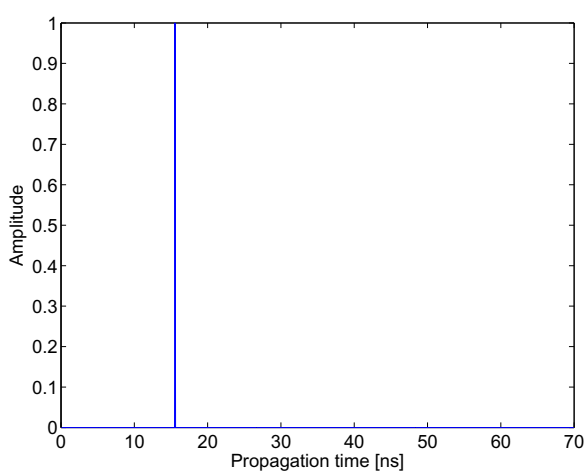

(h)

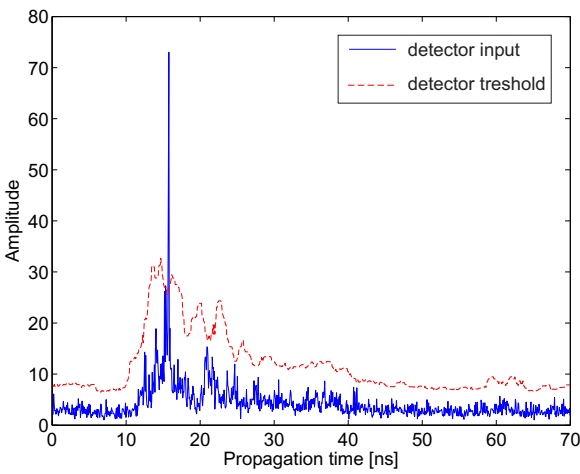

(e)

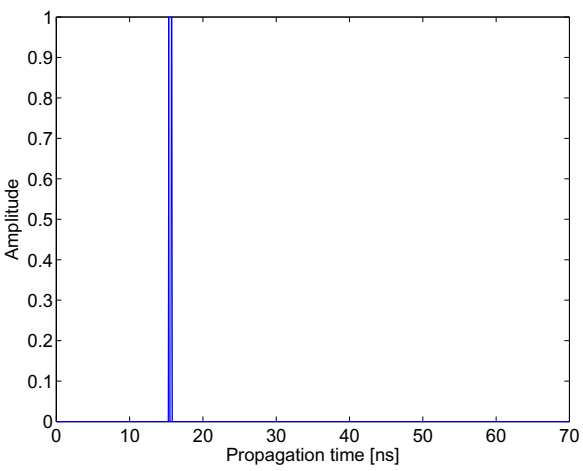

(g)

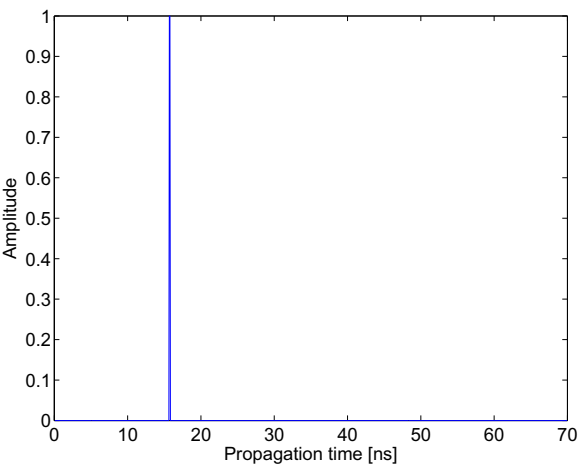

(i)

Fig. 7 The results of selected signal processing phases; phase: (a) background subtraction, (b) weak signal enhancement, (c) the estimates of the power spectrum, (d) and (e) integrated power spectrum along the frequency interval of interest $(<0.1 \mathrm{~Hz} ; 0.7>\mathrm{Hz})$ and the first stage detector treshold for Rx1 and Rx2, (f) and (g) the first stage detector output for Rx1 and Rx2, (h) and (i) the second stage detector output for Rx1 and $\mathrm{Rx} 2$ 


\section{CONCLUSION}

In this paper, a novel method for a static person detection and localization has been presented. The person detection is achieved by the precise monitoring of his respiration movement i.e. uplift and drop of his/her chest.

The main goal of this paper is to point out the performance of the proposed method using Welch periodogram in combination with the two stage detector even when lowcost antennas are being used. All the results were achieved by processing of the radar data collected during the laboratory measurement. To emulate the conditions similar to the real life, no additional signal amplifiers were used. Despite of these conditions, monitored target is localized correctly using both antenna types.

The obtained results indicate, that low-cost compact antipodal Vivaldi antennas can be used for static person localization in conditions similar to laboratory. Besides, a usage of compact antennas in real-life applications is considerably better because of their compact dimensions, low weight and easy manipulation. Therefore, it can be concluded that lowcost compact antipodal Vivaldi antennas could be used for the static person localization under simple scenarios (e.g. line-of-sight scenarios).

\section{ACKNOWLEDGEMENT}

The work was supported by the Slovak Research and Development Agency under the contract No. APVV-0404-12.

\section{REFERENCES}

[1] SACHS, J. - HERRMANN, R.: M-sequence-based ultra-wideband sensor network for vitality monitoring of elders at home, Radar, Sonar \& Navigation, IET, Vol. 9, No. 2, pp. 125-137, 2015.

[2] ROVŇÁKOVÁ, J. - ŠVECOVÁ, M. - KOCUR, D. NGUYEN, T. T. - SACHS, J.: Signal processing for through wall moving target tracking by M-sequence UWB radar, in Radioelektronika, 2008 18th International Conference, IEEE, 2008, pp. 1-4.

[3] LAZARO, A. - GIRBAU, D. - VILLARINO, R.: Analysis of vital signs monitoring using an IR-UWB radar, Progress In Electromagnetics Research, Vol. 100, pp. 265-284, 2010.

[4] SACHS, J.: Handbook of Ultra-wideband Shortrange Sensing: Theory, Sensors, Applications, John Wiley \& Sons, 2013.

[5] ZAIKOV, E. - SACHS, J.: UWB radar for detection and localization of trapped people, INTECH Open Access Publisher, 2010.

[6] ROHLING, H.: Some radar topics: waveform design, range CFAR and target recognition, in Advances in Sensing with Security Applications, pp. 293-322, Springer, 2006.

[7] GAMEC, J. - SCHNEIDER, J. - GAMCOVÁ, M.: Vivaldi Antenna for UWB Sensor Networks, Elek- tronika ir Elektrotechnika, Vol. 22, No. 4, pp. 41-45, 2016.

[8] LAZARO, A. - GIRBAU, D. - VILLARINO, R.: Techniques for clutter suppression in the presence of body movements during the detection of respiratory activity through UWB radars, Sensors, Vol. 14, No. 2, pp. 2595-2618, 2014.

[9] NEZIROVIĆ, A. - YAROVOY, A. G. - LIGTHART, L. P.: Signal processing for improved detection of trapped victims using UWB radar, Geoscience and Remote Sensing, IEEE Transactions on, Vol. 48, No. 4, pp. 2005-2014, 2010.

[10] SACHS, J. - HELBIG, M. - HERRMANN, R. KMEC, M. - SCHILLING, K. - ZAIKOV, E.: Remote vital sign detection for rescue, security, and medical care by ultra-wideband pseudo-noise radar, Ad Hoc Networks, Vol. 13, pp. 42-53, 2014.

[11] URDZÍK, D. - KOCUR, D. - ROVŇÁKOVÁ, J.: Detection of multiple targets with enhancement of weak uwb radar signals for the purposes of through wall surveillance, in Applied Machine Intelligence and Informatics (SAMI), 2012 IEEE 10th International Symposium on, IEEE, 2012, pp. 137-142.

Received June 27, 2016, accepted September 28, 2016

\section{BIOGRAPHIES}

Daniel Novák was born in 1989 in Prešov, Slovakia. He received his Bc. degree in 2011 at Brno University of Technology and his Ing. (M.Sc.) degree at Technical University of Košice at Department of Electronics and Multimedia Communications. His thesis was titled "Moving Person Detection, Localization and Tracking in Complex Environment by UWB Radar Systems". Since September 2013 he has been at this department as a PhD. student. His research interests are UWB radars and signal processing related to them.

Ján Schneider was born in Košice, Slovakia, in 1988. He received Ing. (MSc.) degree in 2013 at the Department of Technology in Electronics, Faculty of Electrical Engineering and Informatics at the Technical University in Košice. His thesis title was "Vibration test in electronics". Since September 2013 he has been at Department of Electronics and Multimedia Communications at Technical University in Košice as $\mathrm{PhD}$. student. His research interests are in antennas, UWB radars and LTCC.

Dušan Kocur was born in 1961 in Košice, Slovakia. He received his Ing. (M.Sc.) and CSc. (Ph.D.) degrees in Radioelectronics from the Faculty of Electrical Engineering, Technical University of Košice, in 1985 and 1990, respectively. Now, he is the full professor at the Department of Electronics and Multimedia Communications of his Alma Mater. His research interests are radar signal processing, UWB technologies and physical layer of wireless communication systems. 